$$
\text { "Herceg" — 2009/2/18 — 23:36 — page } 363-\# 1
$$

\title{
Numerical mathematics with GeoGebra in high school
}

\author{
Dragoslav Herceg and ĐorĐe Herceg
}

\begin{abstract}
We have prepared a suite of motivational examples which illustrate numerical methods for equation solving. Fixed point iteration, Newton's method, secant method and regula falsi method are implemented as GeoGebra tools. Our experience in teaching of numerical mathematics in "Jovan Jovanović Zmaj" high school in Novi Sad is presented. We have tested pupil proficiency in numerical equation solving with and without use of a computer and the results are presented.
\end{abstract}

Key words and phrases: Numerical equation solving, teaching of mathematics, teaching and learning software, CAS, DGS, GeoGebra, Mathematica.

ZDM Subject Classification: N30, U30, U50.

\section{Introduction}

In this paper we present solving equations in GeoGebra. For that purpose, we have developed several GeoGebra tools, as well as a collection of examples, which are available at http://www.im.ns.ac.yu/personal/hercegd/cadgme2007.

Numerical equation solving in high school is a part of the standard curriculum. It is taught in 14 lessons, which cover the following units:

- Localization of roots

- Approximation of a root

- Bisection method

- Newton-Raphson method

- Secant method and regula falsi method

Copyright (c) 2008 by University of Debrecen 


$$
\text { "Herceg" — 2009/2/18 — 23:36 — page } 364 \text { — \#2 }
$$

- Fixed point iteration

Without a computer, these lessons are presented on a whiteboard and by solving only the basic examples. The teacher must carefully choose the problems and solve them completely before class. In the class, the teacher can only present those and similar problems, which can be solved by using well-known properties of elementary mathematical functions.

In order to maximize the positive effects of a lesson, it is essential that the pupils engage actively with their learning. Teaching that accomplishes the maximum effect is interactive, direct and collaborative, and, most of all, interesting. We hope to have achieved this by combining several approaches and tools, which we developed in GeoGebra and Mathematica.

In this paper we present three basic numerical methods for equation solving: bisection method, Newton-Raphson method and regula falsi.

\subsection{The classroom and the software}

The authors teach numerical mathematics in a classroom with 18 PC workstations and one pupil at each workstation. The workstations are networked and have Internet access, as well as USB ports and a CD/DVD drive. The teacher's computer is connected to a data projector and is also used by pupils for demonstrating their work in front of the class. This computer is also a classroom file server. The whiteboard is used for writing down key steps in equation solving. In that way important information is retained, even when the projected picture changes.

The teacher uploads the examples and tools to the file server, thus allowing the pupils to use them during class and exams, when appropriate.

Pupils are allowed to talk to each other and use the Internet, as long as it is about solving the assigned problems. It is important to keep the pupils on track, and sometimes we achieve this by blocking their access to the Internet or specific sites.

We keep record of pupil attendance and classroom activity, which influences their grades. The grading process is transparent, i.e. the pupils are allowed to track their progress and they always know their current standings.

In class we used GeoGebra 2.7 and Mathematica 5. While Mathematica satisfies most of our computing needs, it lacks interactivity, which GeoGebra supplements in more than one way. Besides, GeoGebra is much simpler to use, 
and it is available in many languages, including pupils' native Serbian. Of no lesser importance is the fact that GeoGebra is free, while Mathematica is not.

GeoGebra 3.0, which has appeared only recently, brings some important improvements, such as user-defined tools, which have enabled us to implement tools for numerical methods for equation solving.

\subsection{Localization of roots}

Localization of roots without a computer can be a tedious job. Teachers then usually choose simple examples. For example, to solve the equation $f(x)=0$, where

$$
F(x)=\ln (x+3)-2 \sin x,
$$

we can begin by graphing the function. This graph is not easy to produce without a computer, or at least a calculator. It is much easier to draw a graph of the two functions

$$
g(x)=\ln (x+3) \quad \text { and } \quad h(x)=2 \sin x,
$$

and to look for intersection points, since $f(x)=0$ is equivalent to $g(x)=h(x)$. The pupils can easily graph the elementary functions $g(x)$ and $h(x)$ (Figure 1).

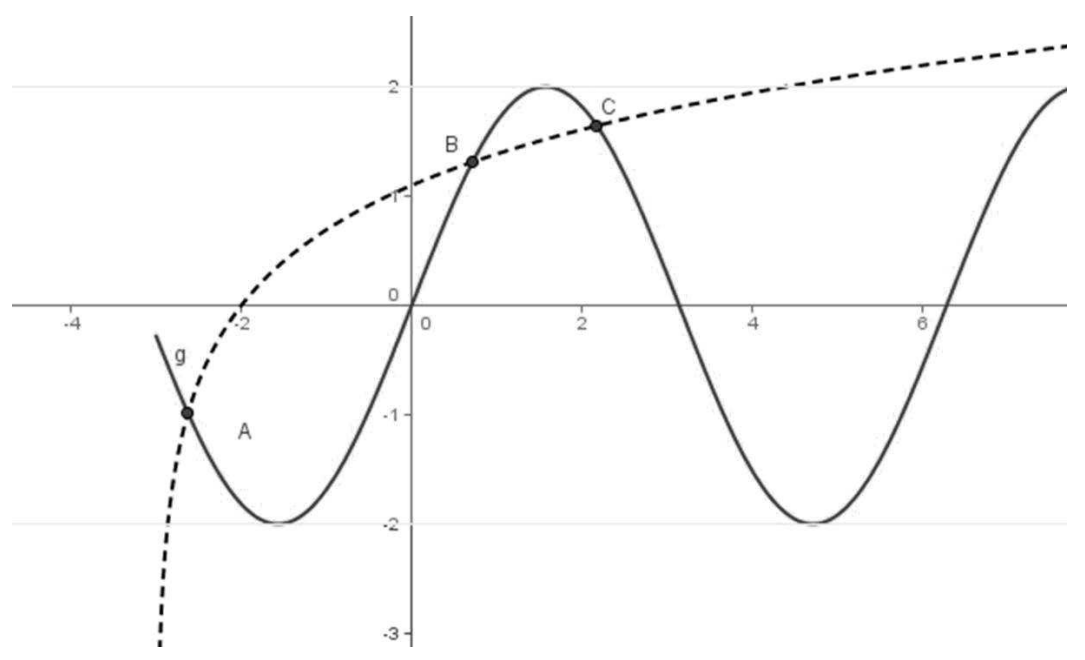

Figure 1

Determining the intervals which contain zeros can be a tedious job. It is also time consuming and requires a rather long series of steps, where possibility 
of error increases with each step. The pupils usually lack the needed skill and experience, and are easily discouraged at the first failure, which quickly leads to a loss of interest on their part.

With a computer, pupils can experiment with varying the interval bounds and find where some of the roots are. To find out how many zeros a function has and where they all are requires a detailed analysis of the function.

In GeoGebra, we can easily find and count the zeros of a function by panning and zooming. GeoGebra enables us to analyze more complex examples, like these:

$$
\begin{gathered}
2 \log x-\frac{1}{2} x+1=0, \quad x-\sin x-\frac{1}{4}=0, \quad x^{2}-20 \sin x=0, \\
2 x \ln x=1, \quad 2^{x}-4 x=0, \quad x^{2}-\sin (\pi x)=0 .
\end{gathered}
$$

Let us consider the equation $x^{2}-20 \sin x=0$. In Figure 2 it is not clear whether there are two or three real roots in the interval $(-8,8)$.

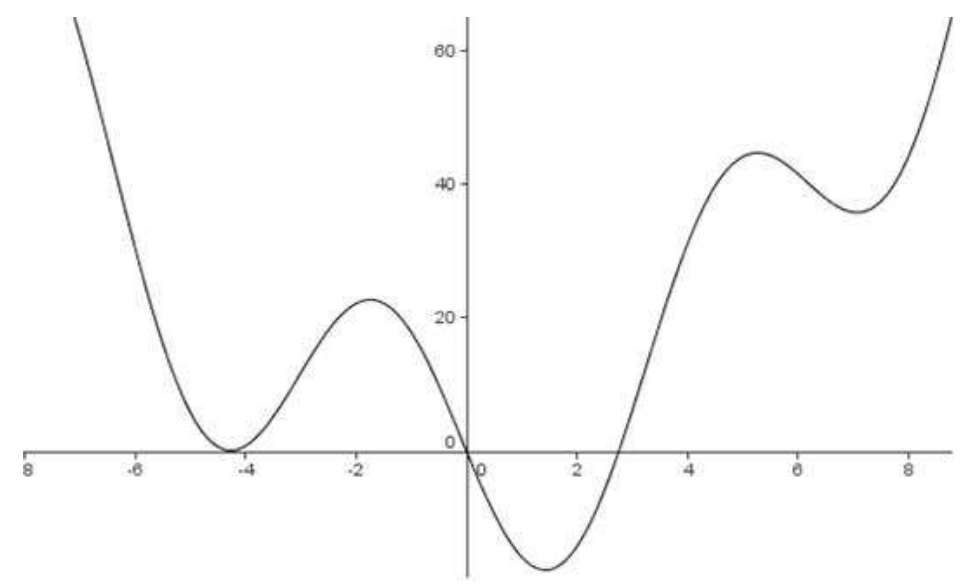

Figure 2. How many zeros does the function have?

By zooming in at the interval $(-5.6,-3.6)$ we see that the curve does not touch the $x$-axis, therefore we conclude that there are only two real roots in the interval $(-8,8)$.

Using GeoGebra, we can look into more complex examples such as:

$$
e^{\sin x}-1=0, \quad 2 \sin (\cos x)=1 .
$$

Simply by moving the function graph by mouse, teacher can modify the problem to get a function with no roots or one with an infinite number of roots. The pupils are encouraged to experiment and investigate new cases and to draw conclusions. 


$$
\text { "Herceg" — 2009/2/18 — 23:36 — page 367 — \#5 }
$$

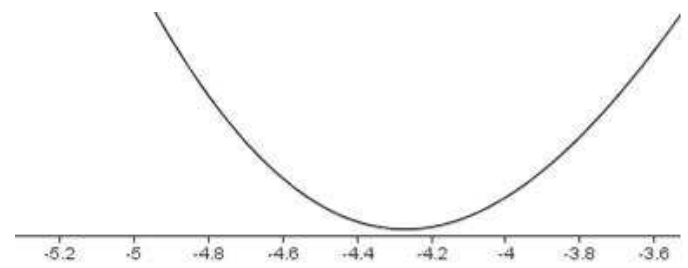

Figure 3. Zooming in at the possible zero

Similarly, we can observe a function which depends on several parameters. For example, the function

$$
f(x)=a e^{b \sin (c x)}+d
$$

has four real parameters $a, b, c$ and $d$. The parameters are controlled by sliders, and by changing their values many interesting exercises are created. We can look for all roots which belong to a given interval, a maximum root, etc.

By zooming in we can read the approximate value for a zero from the intersection point of the graph and the $x$-axis.

The tool "New point" can be used to read the coordinates from the graph, simply by moving the point along the curve. At the intersection of the function with the $x$-axis we can read the approximate value of a real zero of the function. We can then use the intersection tool to set a point there. The point's $x$ coordinate is the approximate value of a zero.

Similarly, a root can be localized at the intersection of two graphs. Here we can emphasize parts of the drawing using different colors and line styles.

We can introduce parameters into the problems, and assign unique tests to each pupil or group of pupils. That way, we can produce an infinite number of examples.

\section{GeoGebra tools for numerical methods}

One of our goals was to facilitate understanding of principles of common iterative methods for numerical equation solving. Recent development of GeoGebra and introduction of user defined tools has made this possible. While in earlier versions of GeoGebra it was neccessary to manually construct tangents, secant lines and midpoints of intervals, now we can simply define a tool and let the pupils 
use it. The pupils who do not understand geometrical interpretation of iterative methods can still use the tools.

We have developed several GeoGebra tools which perform one iteration of the following iterative methods:

- Bisection method,

- Regula-falsi (secant) method,

- Newton-Raphson method.

The tools are based on geometrical representation of these iterative methods. By repeatedly applying a tool at the result of the previous iteration, we can simulate the working of an iterative method.

\subsection{Bisection Method}

One of the first numerical methods developed for finding roots of a nonlinear equation $f(x)=0$ was the bisection method. This is one of the simplest methods and is based on the following theorem.

Theorem. An equation $f(x)=0$, where $f(x)$ is a real continuous function in $[a, b]$, has at least one root between $a$ and $b$ if $f(a) f(b)<0$.

To find a root of $f(x)=0$ using this method, the first thing to do is to find an interval $[a, b]$ such that $f(a) f(b)<0$. Bisect this interval to get a point $(c, f(c))$. Choose one of $a$ or $b$ so that the sign of $f(c)$ is opposite to the sign of ordinate at that point. Use this as the new interval and proceed until you get the root within desired accuracy.

The Bisection tool performs one step of the bisection method. It requires the following input: a function which has real zeros and two points $a$ and $b$ on the $x$-axis. It produces a new point $c$ on the $x$-axis with the coordinates $\left(\frac{a+b}{2}, 0\right)$ and it marks the part of the interval which does not contain the zero with a red line.

EXAMPLE. We shall apply the bisection tool to the equation $2 \sin (\cos x)=1$. First, we need to type in the definition $f(x)=2 \sin (\cos (x))-1$ in GeoGebra's input box. This displays a graph of the function $f(x)$. We obtain the "exact" solution of the equation with the Intersect tool, by finding the intersection point $A$ of the curve with the $x$-axis. Then we select the Bisection tool from the toolbar. In order to perform one step of the bisection method, we need to click on the function in the geometry window, and then click two times on the $x$-axis, 
defining the points $B$ and $C$, which will mark the initial interval (Figure 4). The Bisection tool generates a midpoint $D$, which will be used in the next step.

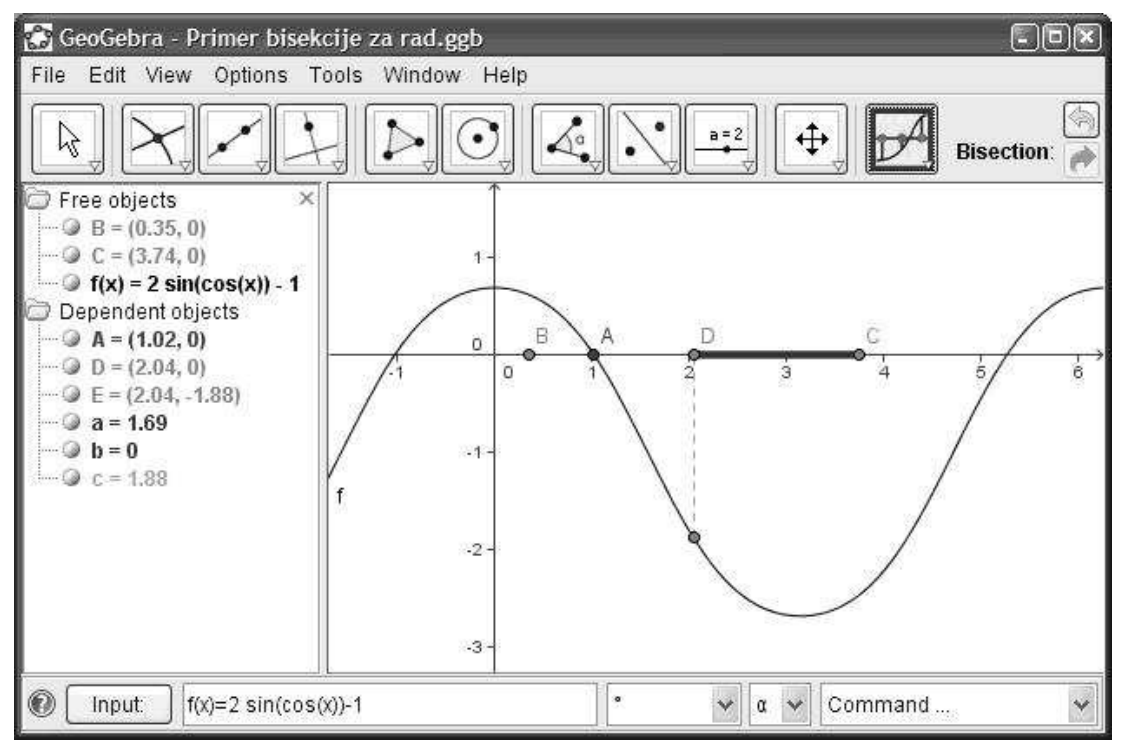

Figure 4

We continue by again clicking on the function, and, this time, by clicking on the points $B$ and $D$, since the interval $[B, D]$ contains the root of the equation.
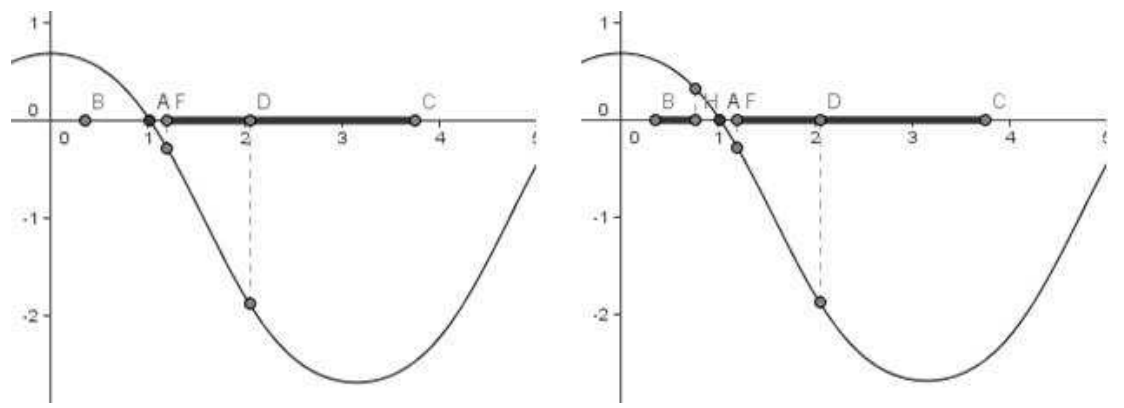

Figure 5. The second and third step of the bisection method

Figure 6 shows the interval which contains a zero after five steps of the bisection method. We need to use the zoom in tool in order to see it. 


$$
\text { "Herceg" — 2009/2/18 - 23:36 — page } 370-\# 8
$$

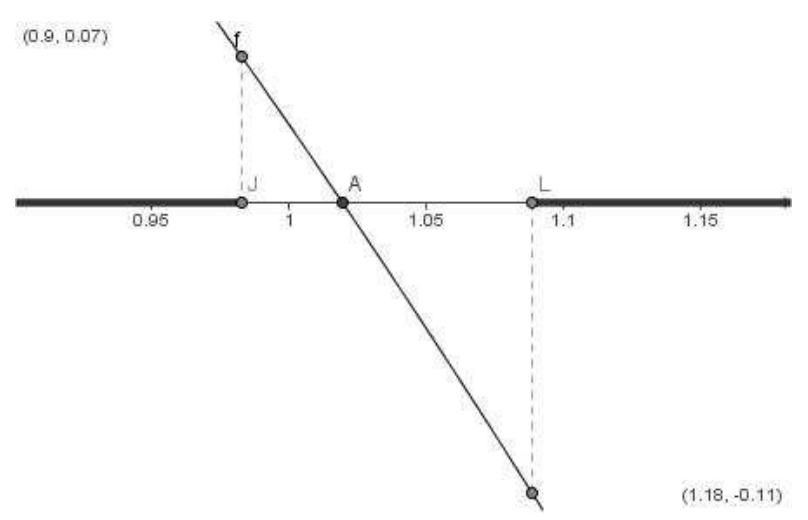

Figure 6. The interval after five iterations

New points, obtained as a result of the bisection tool, are the approximations of the root. In Figure 7, these points are named $D, F, H, J, L$ in the algebra window.

When the interval which contains a zero becomes too small to see, we need to use the zoom in tool in order to get a better view of the interval. Since GeoGebra can display up to five decimal digits, we can get a rather precise approximation of the root. This applies to other numerical methods as well.

\subsection{Newton-Raphson}

The Newton-Raphson method of solving the nonlinear equation $f(x)=0$ is given by the recursive formula

$$
x_{k+1}=x_{k}-\frac{f\left(x_{k}\right)}{f^{\prime}\left(x_{k}\right)} .
$$

One of the drawbacks of the Newton-Raphson method is that the derivative of the function needs to be evaluated for every iteration. With availability of symbolic mathematics software such as Mathematica and GeoGebra, this process has become more convenient. However, it still can be a laborious process.

For Newton-Raphson method only one initial approximation of the root is needed to get the iterative process started to find the root of an equation. This method is based on the principle that if the initial guess of the root of $f(x)=0$ is at $x_{0}$, then if one draws the tangent to the curve at $f\left(x_{0}\right)$, the point $x_{1}$ where the tangent crosses the $x$-axis is an improved estimate of the root (Figure 8). Tangent 


$$
\text { "Herceg" — 2009/2/18 — 23:36 — page } 371 \text { — \#9 }
$$

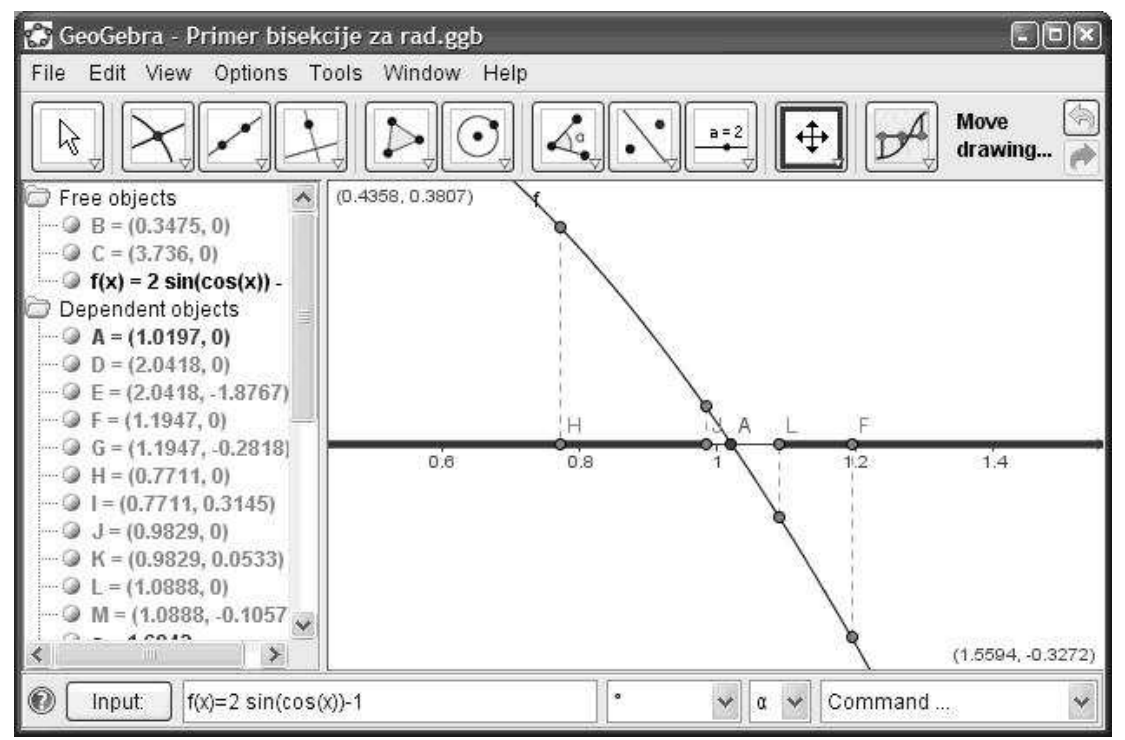

Figure \%. Approximations shown with 5 digits

line is given by

$$
f^{\prime}\left(x_{0}\right)\left(x-x_{0}\right)+f\left(x_{0}\right) .
$$

The solution $x_{1}$ of equation

$$
f^{\prime}\left(x_{0}\right)\left(x-x_{0}\right)+f\left(x_{0}\right)=0
$$

is given by

$$
x_{1}=x_{0}-\frac{f\left(x_{0}\right)}{f^{\prime}\left(x_{0}\right)} .
$$

So starting with an initial guess $x_{0}$ one can find the next approximations $x_{1}, x_{2}, \ldots$, until the root within a desirable tolerance is found.

The steps to apply Newton-Raphson method to find the root of an equation $f(x)=0$ are:

1. Evaluate $f^{\prime}(x)$ symbolically.

2. Use an initial guess of the root, $x_{0}$, to estimate the new value of the root $x_{1}$.

3. Repeat step 2 , using the value $x_{i}$ to obtain a new value $x_{i+1}$.

The NewtonRaphson tool performs one step of the Newton-Raphson method. It requires the following input: a function and an initial point on the $x$-axis. It 


$$
\text { "Herceg" — 2009/2/18 — 23:36 — page 372 — \#10 }
$$

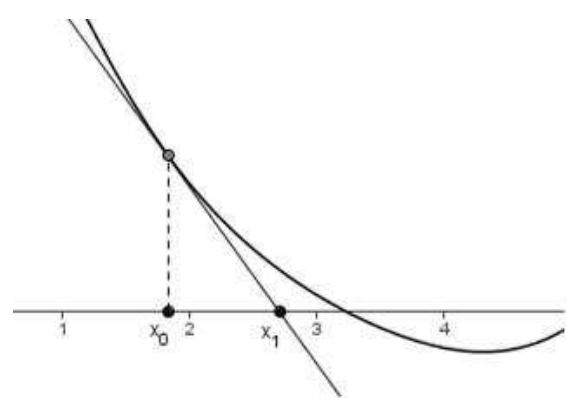

Figure 8. Geometrical representation of the Newton-Raphson method

then produces a tangent to the function and a new approximation in the intersection point of the tangent and the $x$-axis.

EXAMPLE. Let us consider the equation $x+1.5-\sin (x+1.5)+0.06=0$. We start by typing in $f(x)=x+1.5-\sin (x+1.5)+0.06$ into GeoGebra's input box. This displays a graph of the function $f(x)$. We obtain the "exact" solution of the equation by intersecting the curve with the $x$-axis. Now let us select the NewtonRaphson tool from the toolbar. First we click on the function, then on the $x$-axis near the point $(1,0)$. The tool then generates point $C$, which is the next approximation of the root of the equation. Repeating the procedure, we can perform several steps of the Newton-Raphson method (Figure 9).

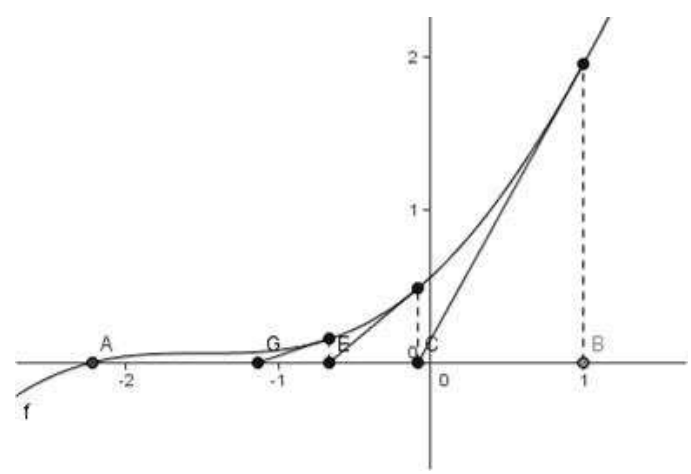

Figure 9. Three steps of the Newton-Raphson method 


\subsection{Regula falsi method}

Methods such as bisection method and the false position method of finding roots of a nonlinear equation $f(x)=0$ require bracketing of the root by two guesses. These methods are always convergent since they are based on reducing the interval between the two guesses to zero in on the root.

In the regula falsi method, we start with two initial points, $x_{0}$ and $x_{1}$, such that $f\left(x_{0}\right) f\left(x_{1}\right)<0$ so that $f(x)=0$ has a solution $\alpha$ between $x_{0}$ and $x_{1}$. We assume that $\alpha$ is the unique solution to $f(x)=0$ between $x_{0}$ and $x_{1}$. The new approximation $x_{2}$ is the point of intersection of the straight line passing through $\left(x_{0}, f\left(x_{0}\right)\right)$ and $\left(x_{1}, f\left(x_{1}\right)\right)$ with the $x$-axis:

$$
x_{2}=x_{1}-\frac{f\left(x_{1}\right)\left(x_{1}-x_{0}\right)}{f\left(x_{1}\right)-f\left(x_{0}\right)} .
$$

If $f\left(x_{2}\right)=0$ then $\alpha=x_{2}$ and we stop. If $f\left(x_{0}\right) f\left(x_{2}\right)<0$, then we leave $x_{0}$ unchanged and continue to the next iteration; otherwise, we set $x_{0}=x_{1}$ and continue to the next iteration in the same way.

In case $f^{\prime}$ and $f^{\prime \prime}$ have fixed signs in an interval containing $\alpha$, which is the situation of interest to us here, the point $x_{0}$ ultimately remains fixed. Therefore, in such a case, the regula falsi method becomes a fixed-point method at some point during the iteration process. Without loss of generality, we will assume that $x_{0}$ remains fixed. In this case the regula falsi method is given by

$$
x_{k+1}=x_{k}-\frac{f\left(x_{k}\right)\left(x_{k}-x_{0}\right)}{f\left(x_{k}\right)-f\left(x_{0}\right)} .
$$

The Secant tool performs one step of the regula falsi method. It requires the following input: a function which has real zeros and two points on the $x$-axis. The tool produces a secant line between the given points and an intersection point with the $x$-axis. It also generates the point $x_{k+1}$, which is used as a starting point for a new iteration.

EXAMPLE. Figure 10 shows three steps of the regula falsi method applied to the equation $e^{\sin (x-3)}-0.5 x=0$. The Secant tool is used similarly to the Bisection and NewtonRaphson tools. Point $A$ is the root of the equation. Points $B$ and $C$ determine the initial interval. 


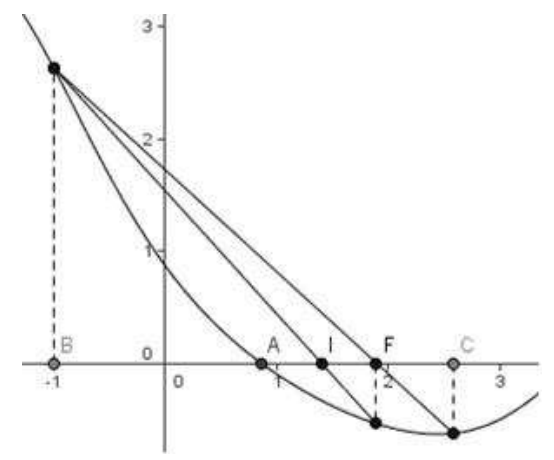

Figure 10. First three steps of the regula falsi method

\section{Testing}

We tested pupil proficiency in numerical equation solving with and without use of GeoGebra. All tests were performed in regular classes during five consecutive school years.

The tests consisted of textbook exercises which were only slightly modified. Grading method was the same during all five years. The first three generations of pupils were taught in Mathematica only, while the last two generations were extensively taught in GeoGebra and Mathematica. It is worth mentioning that the pupils were keen to use both GeoGebra and Mathematica, especially GeoGebra as it is simple to use for root localization. The students were allowed to use computers during the tests, with one exception in 2006/07, which will be discussed later.

The generations of 2005/06 and 2006/07 were divided into two groups. Table 1 shows grade average after testing in numerical equation solving (1-worst, 5-best). Obviously the test scores are better in the last two generations.

\section{Table 1}

\begin{tabular}{|l|c|c|c|c|c|}
\hline School year & $2002 / 03$ & $2003 / 04$ & $2004 / 05$ & $2005 / 06$ & $2006 / 07$ \\
\hline Software used & Mathematica & Mathematica & Mathematica & $\begin{array}{c}\text { Mathematica } \\
\text { GeoGebra }\end{array}$ & $\begin{array}{c}\text { Mathematica } \\
\text { GeoGebra }\end{array}$ \\
\hline Number of pupils & 24 & 22 & 13 & 30 & 28 \\
\hline Grade average & 3.41667 & 3.54545 & 3.23077 & 4.03333 & 4.17857 \\
\hline $\begin{array}{l}\text { Standard } \\
\text { deviation }\end{array}$ & 0,928611 & 0,738549 & 1,48064 & 1.18855 & 1.15642 \\
\hline
\end{tabular}


We have run $t$-tests to pair wise compare the mean values of the samples and to verify that there exists a statistically significant difference between the mean values.

Table 2 shows the obtained confidence intervals for pairs of samples. It should be mentioned that the confidence level is 95\% except for the pair 2005/06 and $2003 / 04$ where it is $90 \%$. The results in Table 2 show that there is a statistically significant difference between the mean values of the samples, as the confidence intervals do not contain the value zero.

Table 2

\begin{tabular}{|c|c|c|}
\cline { 2 - 3 } \multicolumn{1}{c|}{} & $2005 / 06$ & $2006 / 07$ \\
\hline $2002 / 03$ & {$[0.0224394,1.21089]$} & {$[0.170914,1.35290]$} \\
\hline $2003 / 04$ & {$[0.00620997,0.969548]$} & {$[0.0629044,1.20333]$} \\
\hline $2004 / 05$ & {$[0.00998311,1.59515]$} & {$[0.160817,1.73479]$} \\
\hline
\end{tabular}

Table 3 shows grade average by groups for generations of 2005/06 and 2006/07.

Table 3

\begin{tabular}{|l|c|c|c|c|}
\hline School year & \multicolumn{2}{|c|}{$2005 / 06$} & \multicolumn{2}{c|}{$2006 / 07$} \\
\hline Group & I & II & I & II \\
\hline Number of pupils & 17 & 13 & 14 & 14 \\
\hline Grade average & 4.24 & 3.77 & 4.21 & 4.14 \\
\hline
\end{tabular}

During school year 2006/07 the pupils were given two additional tests in numerical equation solving. The topics were graphical localization of roots and localization of roots of algebraic equations. As the pupils were in two groups, we decided to test two approaches in teaching.

The first group was strongly encouraged to use a computer with GeoGebra and some additional programs which we developed. They were instructed to experiment and try to solve the problems in more than one way. This group was allowed to use computers during the test.

The second group was instructed in a more "classical" way, to try to localize the roots by first studying the theory and then graphing the function. This group was not allowed to use computers during the test, although simple electronic calculators were allowed.

For the second test we switched the groups. Table 4 shows the results from the two tests. It can be easily seen that the group which experimented with a computer with GeoGebra and additional programs, achieved better results. 


$$
\text { "Herceg" — 2009/2/18 — 23:36 — page 376 — \#14 }
$$

Table 4

\begin{tabular}{|l|c|c|c|c|}
\hline Tests & \multicolumn{2}{|c|}{ First test } & \multicolumn{2}{c|}{ Second test } \\
\hline Group & I & II & I & II \\
\hline Number of pupils & 14 & 14 & 14 & 14 \\
\hline Grade average & 4.14286 & 2.64286 & 2.50000 & 3.57143 \\
\hline Standard deviation & 1.09945 & 1.49908 & 1.16024 & 1.08941 \\
\hline Confidence interval & {$[0.478707,2.52129]$} & {$[-1.94576,-0.197099]$} \\
\hline
\end{tabular}

We have run a $t$-test to compare the mean values of the samples which stem from the results of the first and second tests. Since neither of the confidence intervals contains a value of zero, we conclude that there is a statistically significant difference between the mean values at the $95 \%$ confidence level.

Use of computer only is not enough for successful problem solving. It is interesting to note that the pupils which were encouraged to use a computer in one exercise did not use it in another exercise, even if it was allowed, but not encouraged. In the second exercise the pupils had no assistance from the teacher, and they fell back to the conventional approach they were used to. It seems that the pupils expected the teacher to prepare all the steps for each particular exercise.

\section{An example of a test}

We present one test which was used during the school year 2006/07.

Problem 1: Find $a$ such that the interval $(a, a+1)$ contains a positive zero of the function $2 x \ln x-1$.

Problem 2: Let $\phi(x)=x-0.4(2 x \ln x-1)$.

a) Prove that the function $\phi$ maps the interval $[1,2]$ onto itself.

b) Prove that the function $\phi$ is a contraction on the interval $[1,2]$.

Problem 3: Let $f(x)=x^{2}-20 \sin x$.

a) How many zeros does the function $f$ have?

Problem 4: Find $r$ and $R$ such that for each root of the polynomial $p(x)=$ $x^{5}+a x^{4}-b x^{2}+c x-3$ the following holds: $1-r<|x|<1+R$. 
Problem 5: Using the bisection method find one root of the polynomial $p(x)=$ $x^{5}+a x^{4}-b x^{2}+c x-3$ with accuracy of $10^{-3}$.

Note: The value $a$ is the number of letters of your first name, the value $b$ is the number of letters of your last name, $c=a+b$.

\section{Conclusion}

The advantages introduced by a computer are not only in quicker calculation and drawing. The computer does all the tedious work, which leaves the teacher and the pupils with enough time to discuss the problem, try out multiple ideas and approaches to solving, and, finally, compare and analyze them. The method of solving a problem is as important as its solution. Use of a computer is particularly important when working with pupils who have difficulties understanding all the aspects of solving a mathematical problem. They are freed from uninspiring and time-consuming solving by hand, so they have more time to learn the important points.

GeoGebra has revolutionalized the way we teach numerical mathematics. It enabled us to do geometrical constructions and animate them easily. It is simple, powerful, and, most importantly, it is free. Compared to other symbolic mathematics software, such as Mathematica, the price/usability ratio of GeoGebra is without a match.

Testing results show that the pupils who were instructed to use GeoGebra have achieved better scores. However, not all the pupils were independent enough to complete the tests without teacher's help and guidance. This confirms the fact that no mathematical software can replace the teacher.

\section{Acknowledgement}

We thank the anonymous reviewer for her/his useful and constructive remarks. 
"Herceg" — 2009/2/18 — 23:36 — page 378 — \#16

\section{References}

[1] D. Herceg and Đ. Herceg, Numerička Matematika, Stylos, Novi Sad, 2003.

[2] M. Hohenwarter, GeoGebra - educational materials and applications for mathematics teaching, PhD thesis, University of Salzburg, Austria, 2006.

[3] J. Uhl and W. Davis, Is the Mathematics We Do the Mathematics We Teach?, Contemporary Issues in Mathematics Education, MSRI Publications 36 (1999), 67-74.

DRAGOSLAV HERCEG and DORDE HERCEG

DEPARTMENT OF MATHEMATICS AND INFORMATICS

FACULTY OF SCIENCE AND MATHEMATICS

UNIVERSITY OF NOVI SAD

TRG DOSITEJA OBRADOVICA 4

21000 NOVI SAD

SERBIA

E-mail: hercegd@im.ns.ac.yu

E-mail: herceg@im.ns.ac.yu

(Received September, 2007) 